\title{
EMBEDDINGS OF QUATERNION SPACE IN $S^{4}$
}

\author{
ATSUKO KATANAGA and OSAMU SAEKI
}

(Received 16 December 1997; revised 28 April 1998)

Communicated by J. A. Hillman

\begin{abstract}
Consider a (real) projective plane which is topologically locally flatly embedded in $S^{4}$. It is known that it always admits a 2-disk bundle neighborhood, whose boundary is homeomorphic to the quaternion space $Q$, the total space of the nonorientable $S^{1}$-bundle over $R P^{2}$ with Euler number \pm 2 , with fundamental group isomorphic to the quaternion group of order eight. Conversely let $f: Q \rightarrow S^{4}$ be an arbitrary locally flat topological embedding. Then we show that the closure of each connected component of $S^{4}-f(Q)$ is always homeomorphic to the exterior of a topologically locally flatly embedded projective plane in $S^{4}$. We also show that. for a large class of embedded projective planes in $S^{4}$, a pair of exteriors of such embedded projective planes is always realized as the closures of the connected components of $S^{4}-f(Q)$ for some locally flat topological embedding $f: Q \rightarrow S^{4}$.

1991 Mathematics subject classification (Amer. Math. Soc.): primary 57N35; secondary 57N13, 57N50, $57 \mathrm{Q} 45$.
\end{abstract}

\section{Introduction}

Consider a connected 1-dimensional polyhedron embedded in $S^{3}$. (In this paragraph, we work in the piecewise linear category.) Then the boundary of its regular neighborhood in $S^{3}$ is a closed connected orientable surface $F$, and thus one obtains an embedding of $F$ into $S^{3}$. Conversely, let $f: F \rightarrow S^{3}$ be an arbitrary embedding. It follows from the Alexander duality that $S^{3}-f(F)$ consists of two connected components. Then Fox [4] has shown that the closure of each connected component is homeomorphic to the closure of the complement of a regular neighborhood of some connected 1-dimensional polyhedron embedded in $S^{3}$.

In this paper, we consider a 4-dimensional analogue of the above result of Fox in

The second author has been partially supported by the Anglo-Japanese Scientific Exchange Programme, run by the Japan Society for the Promotion of Science and the Royal Society.

(c) 1998 Australian Mathematical Society $0263-6115 / 98 \$ A 2.00+0.00$ 
the topological category as follows. Let $P$ be a topologically locally flatly embedded (real) projective plane in $S^{4}$. Then by [6, Section 9.3], $P$ has always a 2-disk bundle neighborhood $N(P)$, which is unique up to ambient isotopy. It is known that the boundary of $N(P)$ is always homeomorphic to the quaternion space $Q$, which is the total space of the nonorientable $S^{1}$-bundle over $\boldsymbol{R} P^{2}$ with Euler number $\pm 2([14,15])$. Thus we obtain a locally flat topological embedding of $Q$ into $S^{4}$. Recall that $Q$ is homeomorphic to $S^{3} / \tilde{D}_{8}$, where $S^{3}$ is the unit sphere in the quaternionic field $\boldsymbol{H}$ and $\tilde{D}_{8}$ is the quaternion group of order eight. In the terminology of [17, Section 5.2], $Q$ is the Seifert fibered space with Seifert invariants $\left\{-1 ;\left(o_{1}, 0\right) ;(2,1),(2,1),(2,1)\right\}$.

Conversely, let $f: Q \rightarrow S^{4}$ be an arbitrary locally flat topological embedding. It follows from the Alexander duality that $S^{4}-f(Q)$ consists of two connected components. The main result of this paper is the following.

THEOREM 1.1. Let $f: Q \rightarrow S^{4}$ be a locally flat topological embedding of the quaternion space $Q$. Then the closure of each connected component of $S^{4}-f(Q)$ is homeomorphic to the closure of $S^{4}-N(P)$ for some topologically locally flatly embedded projective plane $P$ in $S^{4}$, where $N(P)$ denotes a 2-disk bundle neighborhood of $P$ in $S^{4}$.

The above theorem gives a positive answer to Yamada's problem [23, Section 1] in the topological category. Note that a similar result for $S^{1} \times S^{2}$ instead of $Q$ has been obtained in $[13,20]$.

In the second part of the paper, we give an existence theorem of embeddings of $Q$ into $S^{4}$ as follows. Let $E_{i}(i=1,2)$ be the closure of $S^{4}-N\left(P_{i}\right)$, where $P_{i}$ are topologically locally flatly embedded projective planes in $S^{4}$. We consider the following problem: does there exist a locally flat topological embedding $f$ : $Q \rightarrow S^{4}$ such that the closures of the two connected components of $S^{4}-f(Q)$ are homeomorphic to $E_{1}$ and $E_{2}$ ? The second result of this paper is an affirmative answer to this question for a large class of embedded projective planes (see Corollary 3.3); for example, if either $P_{1}$ or $P_{2}$ is the connected sum of the standardly embedded projective plane in $S^{4}$ (see $[12,19]$ ) with a locally flat 2 -knot (see [7-9]), then the answer is affirmative.

The paper is organized as follows. In Section 2, we prove Theorem 1.1. The idea of the proof is to find an appropriate self-homeomorphism $h$ of $Q$ such that the closed 4-manifold obtained by gluing the closure of a component of $S^{4}-f(Q)$ and the 2-disk bundle over $\boldsymbol{R} P^{2}$ by using $h$ is homeomorphic to $S^{4}$. This will be done by using the topological characterization of the 4-sphere, which is due to Freedman [5]. In Section 3, we prove the existence result Corollary 3.3. In Section 4, we discuss related problems in dimension four which are natural generalizations of Fox's problem in dimension three. 
Throughout the paper, we work in the topological category unless otherwise indicated. The symbol " $\approx$ " denotes a homeomorphism between topological spaces

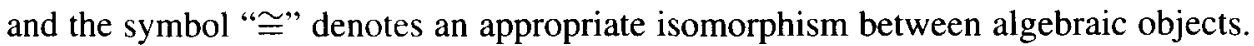
Homology and cohomology groups are always with integral coefficients.

The authors would like to express their sincere gratitude to Yuichi Yamada and Masakazu Teragaito for stimulating discussions and invaluable advice. They also would like to thank Takao Matumoto for his constant encouragement.

\section{Proof of Theorem 1.1}

Let $Q$ be the quaternion space, which is the total space of the nonorientable $S^{1}$ bundle over $R P^{2}$ with Euler number \pm 2 . Let $a$ be an element of $\pi_{1}(Q)$ which corresponds to a fiber and $b$ an element which corresponds to a section over the center circle of a Möbius band embedded in $R P^{2}$. Then it is known that $\pi_{1}(Q)$ has the presentation $\left\langle a, b \mid a^{2}=b^{2}=(a b)^{2}\right\rangle$ (see [18]).

Recall that the outerautomorphism group of $\pi_{1}(Q)$ consists of six elements corresponding to the following automorphisms:

$$
\begin{array}{lll}
\gamma_{0}: & a \mapsto a, & b \mapsto b, \\
\gamma_{1}: & a \mapsto a, & b \mapsto a b, \\
\gamma_{2}: & a \mapsto a b, & b \mapsto a, \\
\gamma_{3}: & a \mapsto b, & b \mapsto a, \\
\gamma_{4}: & a \mapsto a b, & b \mapsto b, \\
\gamma_{5}: & a \mapsto b, & b \mapsto a b .
\end{array}
$$

Let $\mathscr{M}(Q)$ denote the mapping class group of $Q$; more precisely, $\mathscr{M}(Q)$ is the group of isotopy classes of orientation preserving self-homeomorphisms of $Q$. Then Price $[18]$ has shown the following theorem.

THEOREM 2.1. The mapping class group $\mathscr{M}(Q)$ is isomorphic to the outerautomorphism group of $\pi_{1}(Q)$, where the isomorphism is induced by the correspondence which associates the induced automorphism to each self-homeomorphism.

Let us begin the proof of Theorem 1.1. Let $W_{i}(i=1,2)$ denote the closures of the connected components of $S^{4}-f(Q)$. In the following, we identify $\partial W_{1}, \partial W_{2}$ and $\partial N\left(\boldsymbol{R} P^{2}\right)$ with $Q$, where $N\left(\boldsymbol{R} P^{2}\right)$ is the nonorientable 2-disk bundle over $\boldsymbol{R} P^{2}$ with Euler number \pm 2 . We will show that there exists a homeomorphism $h_{i}: \partial N\left(\boldsymbol{R} P^{2}\right) \approx$ $Q \rightarrow Q \approx \partial W_{i}(i=1,2)$ such that the closed 4-manifold $W_{i} \cup_{h_{i}} N\left(\boldsymbol{R} P^{2}\right)$ obtained by gluing $W_{i}$ and $N\left(\boldsymbol{R} P^{2}\right)$ by using $h_{i}$ is homeomorphic to $S^{4}$. 
For the moment, let $h_{i}: Q \rightarrow Q$ be an arbitrary homeomorphism and set $X_{i}=$ $W_{i} \cup_{h_{i}} N\left(\boldsymbol{R} P^{2}\right)$. Since the Euler characteristic of $X_{i}$ is equal to $2, X_{i}$ is a homotopy 4 -sphere if and only if it is simply connected. Thus, by virtue of Freedman's solution of the 4-dimensional Poincaré conjecture in the topological category [5], we have only to show that $X_{i}$ is simply connected for some $h_{i}$.

We need the following lemma.

LEMMA 2.2. $H_{1}\left(W_{i}\right) \cong Z_{2}$ for $i=1,2$.

PROOF. By Alexander duality and Poincaré duality, we have

$$
H_{1}\left(W_{1}\right) \oplus H_{1}\left(W_{2}\right) \cong H_{1}\left(S^{4}-f(Q)\right) \cong H^{2}(Q) \cong H_{1}(Q) \cong Z_{2} \oplus Z_{2} .
$$

By a similar argument, we have $H_{2}\left(W_{1}\right)=0=H_{2}\left(W_{2}\right)$. Suppose that $H_{1}\left(W_{2}\right)=0$. Then by Alexander duality and the universal coefficient theorem, we have

$$
H_{1}\left(W_{1}\right) \cong H^{2}\left(W_{2}\right) \cong \operatorname{Hom}\left(H_{2}\left(W_{2}\right), Z\right) \oplus \operatorname{Tor}\left(H_{1}\left(W_{2}\right), Z\right)=0,
$$

which contradicts (1). By a similar argument, we also see that $H_{1}\left(W_{1}\right)$ is not zero. Hence, by (1), we have $H_{1}\left(W_{1}\right) \cong H_{1}\left(W_{2}\right) \cong Z_{2}$. This completes the proof.

Consider $X_{1}$. (The argument for $X_{2}$ is exactly the same.) By the definition of $W_{1}$ and $W_{2}$, there exists a homeomorphism $g: Q \approx \partial W_{2} \rightarrow \partial W_{1} \approx Q$ such that $W_{1} \cup_{g} W_{2} \approx S^{4}$. Note that $H_{1}\left(W_{i}\right) \cong Z_{2}$ by the above lemma and that $\pi_{1}\left(N\left(\boldsymbol{R} P^{2}\right)\right) \cong$ $H_{1}\left(N\left(R P^{2}\right)\right) \cong Z_{2}$.

Consider Figure 1 , where id, $\mathrm{id}_{1}$ and $\mathrm{id}_{2}$ are the identity maps, $i_{1}, i_{2}, j, w_{0}, w_{1}$, $w_{2}, \bar{w}_{0}, \bar{w}_{1}$ and $\bar{w}_{2}$ are the inclusion maps, and $k=\beta \circ \alpha$ is the composite of the Hurewicz map $\alpha: \pi_{1}\left(W_{2}\right) \rightarrow H_{1}\left(W_{2}\right)$ and an isomorphism $\beta: H_{1}\left(W_{2}\right) \cong Z_{2} \rightarrow$ $Z_{2} \cong \pi_{1}\left(N\left(R P^{2}\right)\right)$.

Set $\phi_{0}=\bar{w}_{0 *} \circ\left(\left(h_{1 *}\right)^{-1} \circ g_{*}\right)=\bar{w}_{1 *} \circ i_{1 *} \circ h_{1 *} \circ\left(\left(h_{1 *}\right)^{-1} \circ g_{*}\right)=\bar{w}_{2_{*}} \circ j_{*} \circ \mathrm{id}_{2} \circ$ $\left(\left(h_{1 *}\right)^{-1} \circ g_{*}\right), \phi_{1}=\bar{w}_{1 *} \circ$ id, and $\phi_{2}=\bar{w}_{2 *} \circ k$. If the commutativity $\phi_{0}=\phi_{1} \circ i_{1 *} \circ g_{*}=$ $\phi_{2} \circ i_{2 *} \circ \mathrm{id}_{1}$ holds, then there exists a unique homomorphism $\lambda: \pi_{1}\left(S^{4}\right) \rightarrow \pi_{1}\left(X_{1}\right)$ such that $\phi_{i}=\lambda \circ w_{i *}(i=0,1,2)$ by van Kampen's theorem (for example, see [3, Chapter V, Section 3]). Note that then $\lambda$ is surjective, since so are id and $k$. Since $\pi_{1}\left(S^{4}\right)$ is trivial, it will follow that $\pi_{1}\left(X_{1}\right)$ is also trivial. So we have only to show that there exists a self-homeomorphism $h_{1}$ of $Q$ for which the above commutativity holds.

Since $\phi_{0}=\phi_{1} \circ i_{1 *} \circ g_{*}$ is clear, we have only to consider the commutativity $\phi_{0}=\phi_{2} \circ i_{2 *} \circ \mathrm{id}_{1}$. Recall that $\phi_{0}=\bar{w}_{2 *} \circ j_{*} \circ \mathrm{id}_{2} \circ\left(\left(h_{1 *}\right)^{-1} \circ g_{*}\right)$ and $\phi_{2}=\bar{w}_{2 *} \circ k$. If the commutativity $k \circ i_{2 *} \circ \mathrm{id}_{1}=j_{*} \circ \mathrm{id}_{2} \circ\left(\left(h_{1 *}\right)^{-1} \circ g_{*}\right)$ holds, then the required commutativity follows. Thus we have only to show the existence of a homeomorphism $h_{1}: Q \rightarrow Q$ such that $k \circ i_{2 *}=j_{*} \circ\left(\left(h_{1 *}\right)^{-1} \circ g_{*}\right)$. 


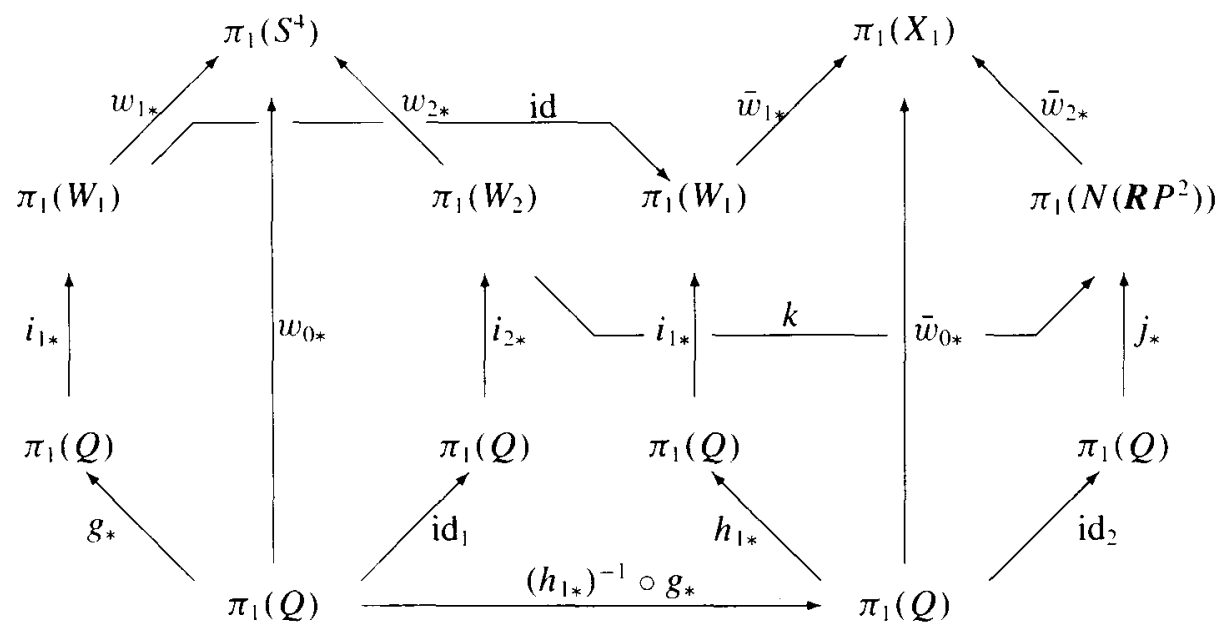

FIGURE 1

Recall that, by Theorem 2.1, every automorphism of $\pi_{1}(Q)$ is realized by an orientation preserving self-homeomorphism. If there exists an automorphism $\psi$ : $\pi_{1}(Q) \rightarrow \pi_{1}(Q)$ such that $j_{*} \circ \psi=k \circ i_{2 *}$, then we can find a self-homeomorphism $h_{1}$ such that $h_{1 *}=g_{*} \circ \psi^{-1}$ and then the required commutativity holds for such an $h_{1}$. It suffices to verify the existence of an automorphism $\psi: \pi_{1}(Q) \rightarrow \pi_{1}(Q)$ such that $j_{*} \circ \psi=k \circ i_{2 *}$ (see Figure 2).

Recall that $\pi_{1}(Q)$ has the presentation of the form $\left\langle a, b \mid a^{2}=b^{2}=(a b)^{2}\right\rangle$. Then by identifying $Q$ with $\partial N\left(\boldsymbol{R} P^{2}\right)$ in a natural manner, we see that $j_{*}(b)$ generates $\pi_{1}\left(N\left(\boldsymbol{R} P^{2}\right)\right) \cong \boldsymbol{Z}_{2}$ and $j_{*}(a)$ is the identity element. Let $c \in H_{1}\left(W_{2}\right) \cong Z_{2}$ denote the generator. Thus we want to find an automorphism $\psi$ which makes Figure 3 commutative, where $\boldsymbol{Z}_{2}\langle x\rangle$ denotes the cyclic group of order two generated by $x$.

Let us consider Figure 4, where $\alpha^{\prime}$ is the Hurewicz homomorphism. Note that this is a commutative figure. Consider the homology exact sequence of the pair $\left(W_{2}, \partial W_{2}\right)=\left(W_{2}, Q\right)$ :

$$
H_{1}(Q) \stackrel{i_{x}}{\longrightarrow} H_{1}\left(W_{2}\right) \longrightarrow H_{1}\left(W_{2}, Q\right) .
$$

By excision, we have $H_{1}\left(W_{2}, Q\right) \cong H_{1}\left(S^{4}, W_{1}\right)=0$. Thus $i_{2 *}: H_{1}(Q) \rightarrow H_{1}\left(W_{2}\right)$ is surjective. Therefore, in Figure $4, \alpha \circ i_{2 *}$ is also surjective. Thus we have the following three possibilities for $\alpha \circ i_{2 *}: \pi_{1}(Q) \rightarrow H_{1}\left(W_{2}\right)$ :

(1) $a \longmapsto c, \quad b \longmapsto 1$,

(2) $a \longmapsto 1, b \longmapsto c$.

(3) $a \longmapsto c, \quad b \longmapsto c$. 


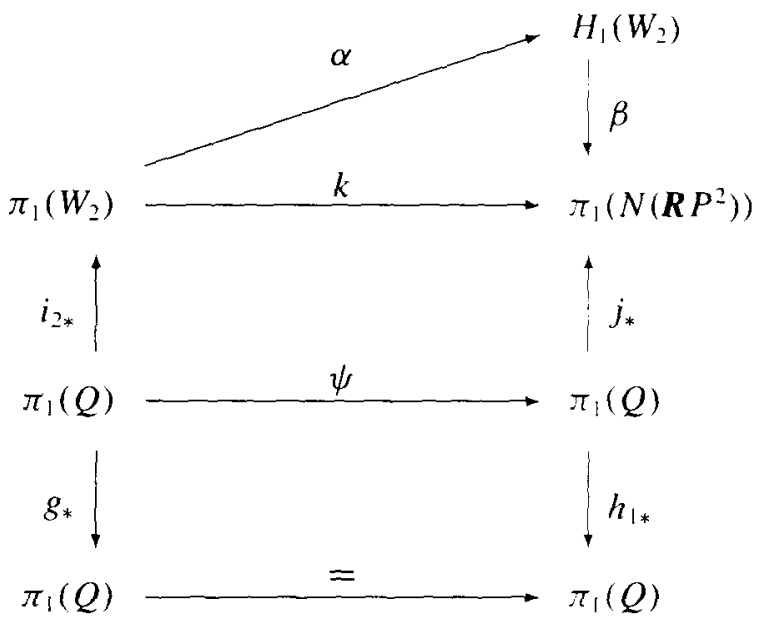

Figure 2

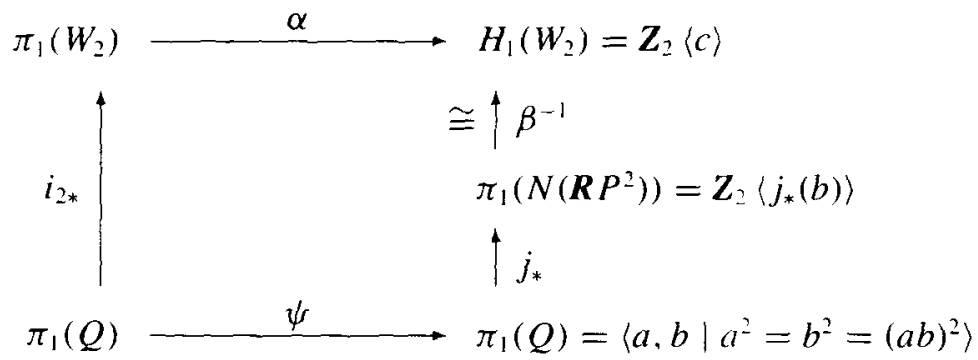

Figure 3

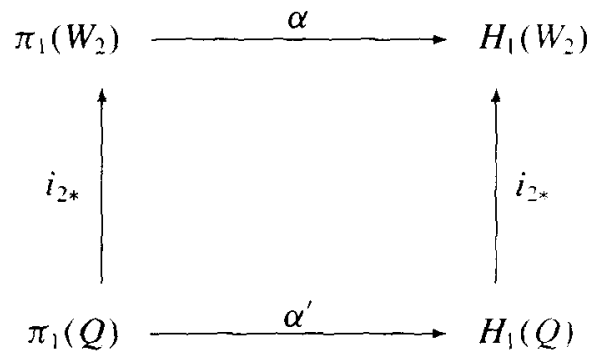

Figure 4 
where $1 \in H_{1}\left(W_{2}\right)=Z_{2}\langle c\rangle$ denotes the identity element. Put

$$
\psi= \begin{cases}\gamma_{2}\left(\text { or } \gamma_{3}\right) & (\text { case }(1)) \\ \gamma_{0}\left(\text { or } \gamma_{1}\right) & (\text { case (2)) } \\ \gamma_{4}\left(\text { or } \gamma_{5}\right) & (\text { case }(3))\end{cases}
$$

where $\gamma_{i}$ are the automorphisms of $\pi_{1}(Q)$ as defined at the beginning of this section. Then it is easy to check that the automorphism $\psi$ makes the required figure (Figure 3) commutative. This completes the proof of Theorem 1.1.

REMARK 2.3. Theorem 1.1 gives an affirmative answer in the topological category to a question posed by Yamada [23, Section 1].

REMARK 2.4. We can also obtain a similar result in the smooth category, provided that we replace $S^{4}$ by homotopy 4 -spheres.

\section{Embeddings with prescribed complements}

Let $P$ be a topologically locally flatly embedded projective plane in $S^{4}$. We denote by $E(P)$ the closure of $S^{4}-N(P)$, where $N(P)$ is a 2-disk bundle neighborhood of $P$ in $S^{4}$ (see [6, Section 9.3]). We call $E(P)$ the exterior of the projective plane $P$. In this section, we show that, for a large class of embedded projective planes in $S^{4}$, a pair of exteriors of such projective planes is always realized as the closures of the connected components of $S^{+}-f(Q)$ for some embedding $f: Q \rightarrow S^{4}$.

PROPOSITION 3.1. Let $P_{j}(j=1,2)$ be topologically locally flatly embedded projective planes in $S^{4}$. Suppose that the kernel of the homomorphism $i_{j *}: \pi_{1}\left(\partial E\left(P_{j}\right)\right) \rightarrow$ $\pi_{1}\left(E\left(P_{j}\right)\right)$ is of order four either for $j=1$ or for $j=2$, where $i_{j}: \partial E\left(P_{j}\right) \rightarrow E\left(P_{j}\right)$ is the inclusion map $(j=1,2)$. Then there exists a homeomorphism $h: \partial E\left(P_{2}\right) \rightarrow$ $\partial E\left(P_{1}\right)$ such that the closed 4-manifold $E\left(P_{1}\right) \cup_{h} E\left(P_{2}\right)$ obtained by gluing $E\left(P_{1}\right)$ and $E\left(P_{2}\right)$ by using $h$ along their boundaries is homeomorphic to $S^{4}$.

REMARK 3.2. It is easy to show that the order of ker $i_{j *}$ is always equal to one, two or four. When it is equal to one, the order of the element $m \in \pi_{1}\left(E\left(P_{j}\right)\right)$ corresponding to the meridian of $P_{j}$ is equal to four. Otherwise, the order of $m$ is equal to two [19, Section VI].

PROOF OF PRoposition 3.1. Set $E_{j}=E\left(P_{j}\right)(j=1,2)$. Since the Euler characteristic of the closed 4-manifold $M_{h}=E_{1} \cup_{h} E_{2}$ is equal to 2 for any homeomorphism $h$, we have only to show that $M_{h}$ is simply connected for some $h$ by 
virtue of Freedman's result [5]. We assume that the kernel of the homomorphism $i_{1 *}: \pi_{1}\left(\partial E\left(P_{1}\right)\right) \rightarrow \pi_{1}\left(E\left(P_{1}\right)\right)$ is of order four.

In the following, we identify $\partial E_{j}=\partial N\left(P_{j}\right)$ with $Q$ so that the $S^{1}$-bundle structure of $Q$ coincides with that of $\partial N\left(P_{j}\right)$ induced from the $D^{2}$-bundle structure of $N\left(P_{j}\right)$. Recall that $\pi_{1}(Q)$ has the presentation $\left\langle a, b \mid a^{2}=b^{2}=(a b)^{2}\right\rangle$, where $a$ corresponds to a fiber. Since $S^{+}$is simply connected, it is easy to see that $\pi_{1}\left(E_{j}\right)$ is normally generated by $i_{j *}(a)$.

It is an easy exercise to list all the subgroups of $G=\pi_{1}(Q)$ of order four, which are as follows:

$$
G_{a}=\left\{1, a, a^{2}, a^{3}\right\}, G_{b}=\left\{1, b, b^{2}, b^{3}\right\}, G_{a b}=\left\{1, a b,(a b)^{2},(a b)^{3}\right\},
$$

where $1 \in G$ denotes the identity element. By our assumption, ker $i_{1 *}$ must coincide with one of the above subgroups. Note that $\operatorname{ker} i_{j *}$ does not contain $a$, since the homology class in $H_{1}\left(E_{j}\right) \cong Z_{2}$ corresponding to $i_{j *}(a) \in \pi_{1}\left(E_{j}\right)$ does not vanish. Thus ker $i_{1 *}$ must coincide with $G_{b}$ or $G_{a b}$.

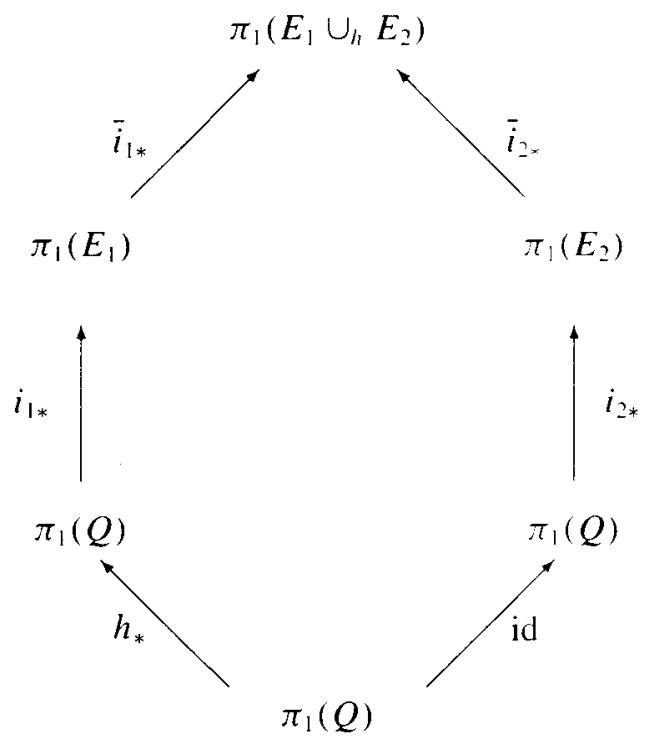

FiguRE 5

Suppose that $\operatorname{ker} i_{1 *}=G_{b}$. We denote by $g_{i}: Q \rightarrow Q(i=0,1, \ldots, 5)$ a selfhomeomorphism of $Q$ which corresponds to the automorphism $\gamma_{i}$ (see Theorem 2.1). Then set $h=g_{3}$. Let us consider Figure 5, which is commutative, where $\bar{i}_{j}(j=1.2)$ are the inclusion maps. By van Kampen's theorem, $\pi_{1}\left(M_{l_{i}}\right)=\pi_{1}\left(E_{1} \cup_{h} E_{2}\right)$ is 
generated by $\bar{i}_{j *}\left(\pi_{1}\left(E_{j}\right)\right)(j=1,2)$ and has relations

$$
\bar{i}_{1 *} \circ i_{1 *} \circ h_{*}(a)=\bar{i}_{2 *} \circ i_{2 *}(a) \quad \text { and } \quad \bar{i}_{1 *} \circ i_{1 *} \circ h_{*}(b)=\bar{i}_{2 *} \circ i_{2 *}(b) \text {. }
$$

By the construction of $h$ together with our assumption on $\operatorname{ker} i_{1 *}$, these two relations are equivalent to

$$
\bar{i}_{2 *} \circ i_{2 *}(a)=1 \quad \text { and } \quad \bar{i}_{1 *} \circ i_{1 *}(a)=\bar{i}_{2 *} \circ i_{2 *}(b) .
$$

Since $i_{2 *}(a)$ normally generates $\pi_{1}\left(E_{2}\right)$, we see that $\bar{i}_{2 *}\left(\pi_{1}\left(E_{2}\right)\right)$ is the trivial group in $\pi_{1}\left(M_{h}\right)$. Thus, by the second relation, we have that $\bar{i}_{1 *} \circ i_{1 *}(a)=1$ in $\pi_{1}\left(M_{h}\right)$. Since $i_{1 *}(a)$ normally generates $\pi_{1}\left(E_{1}\right)$, we see that $\bar{i}_{1 *}\left(\pi_{1}\left(E_{1}\right)\right)$ is also trivial. Hence $\pi_{t}\left(M_{h}\right)$ is the trivial group.

In the other case where ker $i_{1 *}=G_{a b}$, we can use a similar argument to show the required result. This completes the proof.

As a direct consequence of the above proposition, we have the following corollary.

COROLLARY 3.3. Let $P_{i}(j=1.2)$ be topologically locally flatly embedded projective planes in $S^{4}$. Suppose that the kernel of the homomorphism $i_{j *}: \pi_{1}\left(\partial E\left(P_{j}\right)\right) \rightarrow$ $\pi_{1}\left(E\left(P_{j}\right)\right)$ is of order four either for $j=1$ or for $j=2$, where $i_{j}: \partial E\left(P_{j}\right) \rightarrow E\left(P_{j}\right)$ is the inclusion map $(j=1.2)$. Then there exists a locally flat topological embedding $f: Q \rightarrow S^{4}$ such that the closures of the two connected components of $S^{4}-f(Q)$ are homeomorphic to $E\left(P_{1}\right)$ and $E\left(P_{2}\right)$.

REMARK 3.4. We do not know if, in Proposition 3.1 and Corollary 3.3, the condition on the order of ker $i_{1 *}$ or ker $i_{2 *}$ is necessary or not.

In the following, $P_{0}$ will denote a standardly embedded projective plane in $S^{4}$ (see, for example, $[11,12,15,19.21])$. In fact, by [12], it is characterized by the property that $\pi_{1}\left(S^{4}-P_{0}\right) \cong Z_{2}$. In the following, a topologically locally flatly embedded 2-sphere in $S^{4}$ will be called a 2-knot in $S^{4}$. For a 2 -knot $K$, we denote by $P_{0} \sharp K$ the connected sum of $P_{0}$ and $K$ in $S^{4}$. In other words, $P_{0} \sharp K$ is the embedding constructed from $K$ by replacing a small 2-disk with a standard Möbius band (see [7-9]).

COROLLARY 3.5. Let $P$ and $P^{\prime}$ be topologically locally flatly embedded projective planes in $S^{4}$ such that $P=P_{0} \sharp K$ for a 2-knot $K$ in $S^{4}$. Then there exists a locally flat topological embedding $f: Q \rightarrow S^{4}$ such that the closures of the two connected components of $S^{4}-f(Q)$ are homeomorphic to $E(P)$ and $E\left(P^{\prime}\right)$.

The above corollary follows from the fact, which is easy to prove, that if $P$ is of the form $P=P_{0} \sharp K$ for a $2-\operatorname{knot} K$, then $\operatorname{ker}\left(i_{*}: \pi_{1}(\partial E) \rightarrow \pi_{1}(E)\right)$ is of order four, where $E=E(P)$ and $i: \partial E \rightarrow E$ is the inclusion map. 
REMARK 3.6. In the differentiable category, if each of $P$ and $P^{\prime}$ is the connected sum of $P_{0}$ and a smooth 2 -knot, then a result corresponding to Corollary 3.5 has already been obtained in $[23,24]$.

REMARK 3.7. As far as the authors know, all the known examples of projective planes embedded in $S^{4}$ are the connected sum of a standard projective plane $P_{0}$ with a 2-knot. In fact, there is a conjecture that every projective plane embedded in $S^{4}$ is of this form, which is called the Kinoshita Conjecture (for example, see [7-9] and [19, Section V]). Although this conjecture has not appeared in the literature, it has been known to knot theorists in Japan for many years (for example. see [25]). We have a weaker conjecture that for an embedded projective plane $P$ in $S^{4}$, the kernel of the homomorphism $i_{*}: \pi_{1}(\partial E) \rightarrow \pi_{1}(E)$ is always of order four, where $E=E(P)$ and $i: \partial E \rightarrow E$ is the inclusion map. We also do not know if $P$ is topologically equivalent to the connected sum of $P_{0}$ and a $2-$ knot when ker $i_{*}$ is of order four.

REMARK 3.8. We can obtain results similar to the above also in the smooth category. However, as in Remark 2.4, we should replace $S^{+}$with homotopy 4 -spheres.

Remark 3.9. In Proposition 3.1, if $P_{1}=P_{2}$, then we obtain a twisted double decomposition of $S^{+}$. Compare this observation with the results in $[21,22]$.

\section{Related problems}

In this section, we first work in the piecewise linear category. Let $\Gamma$ and $\Delta$ be compact connected polyhedrons. We suppose that there exist "standard" embeddings $\varphi_{0}: \Gamma \rightarrow S^{4}$ and $\psi_{0}: \Delta \rightarrow S^{4}$ such that $\left.\varphi_{0}(\Gamma) \cap \psi_{0}(\Delta)=\emptyset\right) N_{\Gamma} \cup N_{\lrcorner}=S^{4}$ and $N_{\Gamma} \cap N_{\Delta}=\partial N_{\Gamma}=\partial N_{\Delta}$, where $N_{\Gamma}$ and $N_{\Delta}$ are regular neighborhoods of $\varphi_{0}(\Gamma)$ and $\psi_{0}(\Delta)$ in $S^{4}$ respectively. Set $M=\partial N_{\Gamma}=\partial N_{\lrcorner}$, which is a closed connected orientable 3-manifold.

Note that many examples of such decompositions of $S^{+}$as above are known. We will give explicit examples later in this section.

Problem 4.1. Let $f: M \rightarrow S^{+}$be an arbitrary piecewise linear (locally flat) embedding and $A$ and $B$ the closures of the connected components of $S^{+}-f(M)$. Then, do there exist embeddings $\varphi: \Gamma \rightarrow S^{4}$ and $\psi: \Delta \rightarrow S^{\dagger}$ such that $A$ and $B$ (or $B$ and $A$ ) are homeomorphic to the closures of $S^{+}-N(\varphi(\Gamma))$ and $S^{+}-N(\psi(\Delta)$ ) respectively, where $N(\varphi(\Gamma))$ and $N(\psi(\Delta))$ are regular neighborhoods of $\varphi(\Gamma)$ and $\psi(\Delta)$ in $S^{\dagger}$ respectively?

The above problem can be regarded as a generalization to 4-dimensions of the problem considered by Fox [4]. 
EXAMPLE 4.2. Let $\Gamma$ and $\Delta$ be distinct two points in $S^{4}$. Then $S^{4}$ decomposes as $S^{4}=N_{\Gamma} \cup N_{\Delta}$, where $N_{\Gamma}$ and $N_{\Delta}$ are homeomorphic to the 4-dimensional disk $D^{4}$. Thus $M=S^{3}$ and the above problem is nothing but the Schoenflies conjecture for dimension four, which has not been solved until now. Note that it has been solved in the topological category by Brown $[1,2]$.

EXAMPLE 4.3. Let $\Gamma$ be a circle and $\Delta$ a 2 -sphere. Let $\psi_{0}: \Delta \rightarrow S^{4}$ be the standard embedding and $\varphi_{0}: \Gamma \rightarrow S^{+}-\psi_{0}(\Delta)$ an embedding such that $\varphi_{0}(\Gamma)$ and $\psi_{0}(\Delta)$ have linking number \pm 1 in $S^{4}$. Then $S^{4}$ decomposes as $S^{4}=N_{\Gamma} \cup N_{\Delta}$, where $N_{\Gamma}$ is homeomorphic to $S^{1} \times D^{3}$ and $N_{\Delta}$ is homeomorphic to $S^{2} \times D^{2}$. In this case, we have $M=S^{1} \times S^{2}$. Then Problem 4.1 has been solved for this case in the topological category in $[13,20]$. In fact, every locally flat topological embedding of $S^{1} \times S^{2}$ into $S^{4}$ bounds $D^{2} \times S^{2}$ embedded in $S^{4}$.

EXAMPLE 4.4. Let $\Gamma=\Delta=\boldsymbol{R} P^{2}$ and $\varphi_{0}: \boldsymbol{R} \boldsymbol{P}^{2} \rightarrow S^{4}$ a standard embedding (see, for example, $[11,12,15,19,21])$. In other words, $\varphi_{0}\left(\boldsymbol{R} P^{2}\right)$ is constructed from the standard embedding of $S^{2}$ by replacing a small 2-disk with a standard Möbius band. Then it is well-known that the closure of $S^{4}-N\left(\varphi_{0}\left(\boldsymbol{R} P^{2}\right)\right)$ is also a regular neighborhood of a standardly embedded $\boldsymbol{R} P^{2}$ in $S^{4}$, by which we define $\psi_{0}: \Delta \rightarrow S^{4}$. Thus $S^{4}$ decomposes as $S^{4}=N_{\Gamma} \cup N_{\Delta}$ and in this case $M$ is the quaternion space $Q$. Then our Theorem 1.1 gives an affirmative answer to Problem 4.1 for this case in the topological category.

EXAMPLE 4.5. For an integer $n \geq 2$, let $X_{n}$ be the polyhedron consisting of a circle $S^{1}$ together with a 2-disk $D^{2}$ attached to $S^{1}$ by an $n$-fold covering map $\partial D^{2} \rightarrow S^{1}$. Note that $X_{2}$ is homeomorphic to $R P^{2}$. Set $\Gamma=\Delta=X_{n}$. Then Yamada [21] has shown that there exists a "standard" embedding $\varphi_{0}: \Gamma \rightarrow S^{4}$ such that the closure of the complement of its regular neighborhood is again a regular neighborhood of a "standard" embedding of $X_{n}$ into $S^{4}$, by which we define $\psi_{0}: \Delta \rightarrow S^{4}$. In this case, $M$ is the Seifert fibered 3-manifold with Seifert invariants $\left\{-1 ;\left(o_{1}, 0\right) ;(n, 1),(n, 1),(n, n-1)\right\}$. The authors do not know an answer to Problem 4.1 for this case.

EXAMPLE 4.6. Let $\Gamma$ be the 2-dimensional torus and $\varphi_{0}: \Gamma \rightarrow S^{4}$ the standard embedding, that is, it bounds an embedded $S^{1} \times D^{2}$ in $S^{4}$. The closure of the complement of a regular neighborhood of $\varphi_{0}(\Gamma)$ in $S^{4}$ is known as Montesinos' twin [16], which is denoted by $T u$. In fact, $T w$ is a regular neighborhood of a polyhedron $\Delta$ embedded in $S^{4}$ which consists of two unknotted 2-spheres intersecting each other transversely at two points with distinct signs. We define $\psi_{0}: \Delta \rightarrow S^{4}$ by the inclusion map. Then for the decomposition $S^{4}=N_{\Gamma} \cup N_{\Delta}$ with $N_{\Gamma}=T^{2} \times D^{2}$ and $N_{\Delta}=T w$, the 3-manifold $M$ is the 3-dimensional torus $T^{3}$. The authors do not know an answer to Problem 4.1 for embeddings of $T^{3}$ into $S^{4}$. 
One can construct a lot of examples of embeddings of $T^{3}$ into $S^{4}$ as follows. (The authors are indebted to Masakazu Teragaito for the idea of the construction.) Let $D_{+}$be the upper hemisphere of $S^{+}$(and $D_{-}$the lower hemisphere of $S^{4}$ ), which is homeomorphic to $D^{+}$. Let $D_{\Gamma}$ be the union of two 2-disks which is properly embedded in $D_{+}$such that $\left(D_{+}, D_{\Gamma}\right)$ is homeomorphic to $\left(D^{2},\{2\right.$ points $\left.\}\right) \times D^{2}$. Take an embedded circle $C_{\Delta}$ in $\partial D_{+}$which does not intersect $D_{\Gamma}$ such that the 3-component link $\partial D_{\Gamma} \cup C_{\Delta}$ in $\partial D_{+}$is the Borromean ring (see, for example, [10, the rightmost figure of Figure 5.2, p.14]). Then let $D_{\Delta}$ be a 2-disk properly embedded in $D_{-}$such that $\left(D_{-}, D_{\Delta}\right)$ is homeomorphic to $\left(D^{2},\{\right.$ a point $\left.\}\right) \times D^{2}$. Let $H_{\Gamma}$ and $H_{\Delta}$ be small regular neighborhoods of $D_{\Gamma}$ in $D_{+}$and $D_{\Delta}$ in $D_{-}$respectively. Then it is not difficult to show that $\left(S^{4}, N_{\Gamma}, N_{\Delta}\right)$ is homeomorphic to $\left(D_{+} \cup D \ldots N_{\Gamma}^{\prime}, N_{\Delta}^{\prime}\right)$, where $N_{\Gamma}^{\prime}$ is the closure of $\left(D_{+}-H_{\Gamma}\right) \cup H_{\Delta}$ and $N_{\Delta}^{\prime}$ is the closure of $\left(D-H_{\Delta}\right) \cup H_{\Gamma}$. In fact, $T w$ has a handlebody structure consisting of a 0 -handle, a 1 -handle and two 2-handles, and the union of the 0 -handle and the 1-handle corresponds to the closure of $D_{-}-H_{\Delta}$ and the two 2-handles correspond to $H_{\Gamma}$ (see [16, Section 3]). Furthermore, $T^{2} \times D^{2}$ has a handlebody structure consisting of a 0-handle, two 1-handles and a 2-handle, and the union of the 0 -handle and the two 1-handles corresponds to the closure of $D_{+}-H_{\Gamma}$ and the 2-handle corresponds to $H_{\Delta}$ (see, for example, [10, Example 5.3. p. 14 and pp. 41-42]). Then the standard embedding of $T^{3}$ into $S^{4}$ is nothing but $\partial N_{\Gamma}^{\prime}=\partial N_{\Delta}^{\prime}$. Therefore, replacing $D_{\Gamma} \subset D_{+}$and $D_{\Delta} \subset D_{-}$with arbitrary linked two 2-disks $\tilde{D}_{\Gamma}$ and an arbitrary knotted 2-disk $\tilde{D}_{\Delta}$ respectively, one obtains a more complicated embedding of $T^{3}$. For example, if both $\tilde{D}_{1}$ and $\tilde{D}_{\Delta}$ are "sufficiently nontrivial", then the closures of the connected components of the complement are not homeomorphic to $T^{2} \times D^{2}$ nor $T w$. Note that for embeddings of $T^{3}$ thus constructed. the answer to Problem 4.1 is affirmative.

\section{References}

[1] M. Brown, 'A proof of the generalized Schoenflies theorem'. Bull. Amer. Math. Soc. 66 (1960). $74-76$.

[2] — , 'Locally flat embeddings of topological manifolds'. in: Topology of 3-Manifolds and Related Topics (Prentice-Hall, New Jersey, 1962) pp. 83-91.

[3] R. H. Crowell and R. H. Fox, Introduction to knot theory (Ginn and Comp., Boston. 1963).

[4] R. H. Fox, 'On the embedding of polyhedra in 3-space', Ann. of Math. 49 (1948), 462-470.

[5] M. H. Freedman, 'The topology of four dimensional manifolds', J. Differential Geom. 17 (1982). $357-453$.

[6] M. H. Freedman and F. Quinn, Topology of 4-manifolds (Princeton Univ. Press, Princeton, New Jersey, 1990).

[7] S. Kamada, 'On deform-spun projective planes in 4-sphere obtained from peripheral inverting deformations', in: Algebra and topology (Taejon, 1990), Proc. of KAIST Math. Workshop 5. Korea, 1990, pp. 197-203. 
[8] —_ 'Projective planes in 4-sphere obtained by deform-spinnings', in: Knots 90 (ed. A. Kawauchi) (Walter de Gruyter, Berlin. New York, 1992) pp. 125-132.

[9] S. Kinoshita, 'On the Alexander polynomial of 2-spheres in a 4-sphere', Ann. of Math. 74 (1961), 518-531.

[10] R. C. Kirby, The topology of 4-manifolds, Lect. Notes in Math., 1374 (Springer, Berlin, 1989).

[11] T. Lawson, 'Splitting $S^{4}$ on $\boldsymbol{R} P^{2}$ via the branched cover of $\boldsymbol{C} P^{2}$ over $S^{4}$, Proc. Amer. Math. Soc. 86 (1982), 328-330.

[12] — 'Detecting the standard embeddings of $R P^{2}$ in $S^{4}$, Math. Ann. 267 (1984), 439-448.

[13] L. A. Lucas, O. M. Neto and O. Saeki, 'A generalization of Alexander's torus theorem to higher dimensions and an unknotting theorem for $S^{p} \times S^{4}$ embedded in $S^{p+q+2}$, Kobe J. Math. 13 (1996), 145-165.

[14] W. S. Massey, 'Proof of a conjecture of Whitney', Pacific J. Math. 31 (1969), 143-156.

[15] _ _ 'Imbeddings of projective planes and related manifolds in spheres', Indiana Univ. Math. J. 23 (1974), 791-812.

[16] J. M. Montesinos, 'On twins in the four-sphere I', Quart. J. Math. Oxford 34 (1983), 171-199.

[17] P. Orlik, Seifert manifolds, Lect. Notes in Math., 291 (Springer, Berlin, 1972).

[18] T. M. Price, 'Homeomorphisms of quaternion space and projective planes in four space', J. Austral. Math. Soc. Ser. A 23 (1977). 112-128.

[19] T. M. Price and D. M. Roseman, 'Embeddings of the projective plane in four space', preprint, (Univ. of Iowa, 1975).

[20] J. H. Rubinstein, 'Dehn's lemma and handle decompositions of some 4-manifolds', Pacific J. Math. 86 (1980), 565-569.

[21] Y. Yamada, 'Decomposition of $S^{4}$ as a twisted double of a certain manifold', Tokyo J. Math. 20 (1997), 23-33.

[22] _ _ 'Some Seifert 3-manifolds which decompose $S^{4}$ as a twisted double', in: Knots '96 (ed. S. Suzuki) (World Scientific. 1997) pp. 545-550.

[23] — - 'Decomposition of the four sphere as a union of $\boldsymbol{R} \boldsymbol{P}^{2}$-knot exteriors', in: Proc. Applied Math. Workshop, vol. 8 (eds. G. T. Gin and K. H. Ko, Center for Applied Math., KAIST, Taejon, 1998) pp. 345-351.

[24] Y. Yamada, O. Saeki, M. Teragaito and A. Katanaga, 'Gluck surgery along a 2-sphere in a 4manifold is realized by surgery along a projective plane', Preprint Series, UTMS 97-42, Univ. of Tokyo, 1997.

[25] K. Yoshikawa, 'Surfaces in $\boldsymbol{R}^{4}$ ( (in Japanese), in: Proc. of infinite groups and topology (Osaka, 1986) pp. 76-98.

Institute of Mathematics

University of Tsukuba

Tsukuba-city, Ibaraki 305-8571

Japan

e-mail: fuji@ math.tsukuba.ac.jp
Department of Mathematics

Faculty of Science

Hiroshima University

Higashi-Hiroshima 739-8526

Japan

e-mail: saeki@math.sci.hiroshima-u.ac.jp 